"Mircea cel Batran" Naval Academy Scientific Bulletin, Volume XIX - 2016 - Issue 1

Published by "Mircea cel Batran" Naval Academy Press, Constanta, Romania // The journal is indexed in:

PROQUEST / DOAJ / DRJI / JOURNAL INDEX / I2OR / SCIENCE LIBRARY INDEX / Google Scholar / Crossref /

Academic Keys / ROAD Open Access / OAJI / Academic Resources / Scientific Indexing Services / SCIPIO

\title{
ELECTRIC AND MAGNETIC FIELD MEASUREMENTS FROM A SHIP FOUND IN THE PORT AREA
}

\author{
Gheorghe SAMOILESCU ${ }^{1}$ \\ Florentiu DELIU² \\ Adelina BORDIANU ${ }^{3}$ \\ Serghei RADU ${ }^{4}$ \\ ${ }^{1}$ Captain Professor engineer, Ph.D, "Mircea cel Batran" Naval Academy, Constanta \\ 2Engineer, Ph.D, "Mircea cel Batran" Naval Academy, Constanta \\ ${ }^{3}$ Lecturer engineer Ph D, University "Politehnica" of Bucharest \\ ${ }^{4}$ Marine Chief Engineer, PdD, Stena Crewing
}

\begin{abstract}
In order to conduct electromagnetic field measurements on board a ship four different locations have been used: the upper deck; the aft; the command point- exterior; the navigating bridge - inside. Within each location measurements were performed with different stations located in broadcasting, on different frequency ranges and operating modes (AM - amplitude modulation and FM - frequency modulation), depending on the available sensors. The measurements made on the ship targeted the following frequency ranges: $88-200 \mathrm{MHz}, 200-2500 \mathrm{MHz}$. Measurements carried out on the vessel gaved data on: the electric field $E[V / m]$, for different frequency ranges; the electric field $[d B \mu V / m]$; rate exposure; ER: E2/L2; field strength limit, L [V / m]; measurement error, ER * 1000 [\%o]; electromagnetic power flux density, $P D$ (or S) $[\mu \mathrm{W} / \mathrm{cm} 2]$; total field strength (RMS) $[\mathrm{V} / \mathrm{m}]$; maximum single $[\mathrm{V} / \mathrm{m}]$.
\end{abstract}

Key words: electric field, magnetic field measurements, exposure rate, flux density

\section{Introduction}

The measuring equipment used in order to perform field measurements consists of::

1. Portable spectrum analyzer with accessories and specialized software - R\&S FSP13 (1164.4391.13)-Spectrum analyzer $9 \mathrm{kHz}$ $13 \mathrm{GHz},-140-+30 \mathrm{dBm}$, RBW $10 \mathrm{~Hz}-10 \mathrm{MHz}$, TFT color display; R\&S FSP-B1 (1129.7998.02)Rugged case with variable carrying handle for all FSP models; R\&S FSP-B16 (1129.8042.03)-LAN interface $10 / 100$ base $T$ for FSP with Nr.1164.4391.XX; R\&S FSP-B9 (1129.6991.02)Tracking generator for FSP, $9 \mathrm{kHz}-3 \mathrm{GHz}$, I/Q modulator; R\&S FSP-B28 (1162.9915.02)-Trigger port for FSP; for indication of trigger conditions (necessary for operation with TS-EMF); R\&S FSP-B30 (1155.1158.02)-DC power supply 12 $28 \mathrm{~V}$ for FSP spectrum analyzer and ESPI test receiver; R\&S FSP-B31 (1155.1258.02)-NiMH battery pack + charger for FSP, requires FSP-B1 and FSP-B30; R\&S FS-K9 (1157.3006.02) Measurements with NRP power sensors NRP-Z11 / Z21 / Z22 / Z23 / Z24 / Z51 / Z55 / Z91 requires NRP-Z3 or NRP-Z4; R\&S NRP-Z4 (1146.8001.02) USB adapter (passive) for NRP-Z sensors powered via USB ; R\&S NRP-Z22 (1137.7506.02) Average power sensor, $10 \mathrm{MHz}-18 \mathrm{GHz}, 2 \mathrm{nW}$ $2 \mathrm{~W}$ for universal use; RF Cable Huber \& Suhner (23005046) 13GHz, 1m long, $N$ male connectors.

2. TS-EMF system - portable izotropic antenna - R\&S TS-EMF (1158.9295.03)-Portable EMF measurement system $30 \mathrm{MHz}-3 \mathrm{GHz}, 1 \mathrm{mV} / \mathrm{m}$ $100 \mathrm{~V} / \mathrm{m}$ (without FSH 3, laptop and carrier bag);

DOI: 10.21279/1454-864X-16-I1-050

(c) 2015. This work is licensed under the Creative Commons Attribution-Noncommercial-Share Alike 4.0 License.
R\&S TS-EMFZ2 (1166.5708.02)-Cable set for TSEMF; R\&S TS-EMF-O3 (1101.8477.03)-EMCtripod forTS-EMF; R\&S RNB (0272.4910.50)Termination (50 Ohm,1 W,DC-4 GHz,N-male) [1]. Measurements data - tabular and graphical representation

The measurements were conducted in three locations within the port for four different points from the ship: the upper deck (1); the aft (2); the command point- exterior (3); the navigating bridge - inside (4). Different specialty papers were taken into account [2-10].

Representation in a fixed set of coordination (on the aft deck).

The chart presented in figure 1 is based on the performed measurements - see Table 1.

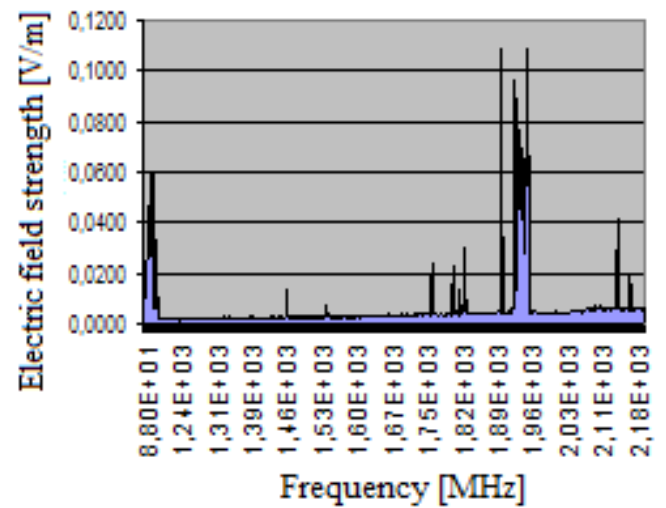

Fig. 1. Ambient noise measurements at 09.43 (background 1, point 1) 
"Mircea cel Batran" Naval Academy Scientific Bulletin, Volume XIX - 2016 - Issue 1

Published by "Mircea cel Batran" Naval Academy Press, Constanta, Romania // The journal is indexed in:

PROQUEST / DOAJ / DRJI / JOURNAL INDEX / I2OR / SCIENCE LIBRARY INDEX / Google Scholar / Crossref /

Academic Keys I ROAD Open Access / OAJI / Academic Resources / Scientific Indexing Services / SCIPIO

Table 1

\begin{tabular}{|l|l|l|}
\hline $\begin{array}{l}\text { Frequency } \\
\mathrm{MHz}\end{array}$ & $\begin{array}{l}\text { Field Strength } \\
\mathrm{V} / \mathrm{m}\end{array}$ & $\begin{array}{l}\text { Power Density } \\
\mu \mathrm{W} / \mathrm{cm} 2\end{array}$ \\
\hline $1,91 \mathrm{E}+03$ & 0,1097 & 0,0032 \\
\hline $1,96 \mathrm{E}+03$ & 0,1087 & 0,0031 \\
\hline $1,90 \mathrm{E}+03$ & 0,1044 & 0,0029 \\
\hline $1,93 \mathrm{E}+03$ & 0,0962 & 0,0025 \\
\hline $1,96 \mathrm{E}+03$ & 0,0913 & 0,0022 \\
\hline $1,94 \mathrm{E}+03$ & 0,0897 & 0,0021 \\
\hline $1,96 \mathrm{E}+03$ & 0,0766 & 0,0016 \\
\hline $1,96 \mathrm{E}+03$ & 0,0739 & 0,0014 \\
\hline $1,93 \mathrm{E}+03$ & 0,0733 & 0,0014 \\
\hline $1,95 \mathrm{E}+03$ & 0,0717 & 0,0014 \\
\hline $1,94 \mathrm{E}+03$ & 0,0709 & 0,0013 \\
\hline $1,95 \mathrm{E}+03$ & 0,0703 & 0,0013 \\
\hline $1,94 \mathrm{E}+03$ & 0,0689 & 0,0013 \\
\hline $1,94 \mathrm{E}+03$ & 0,0676 & 0,0012 \\
\hline $1,94 \mathrm{E}+03$ & 0,0643 & 0,0011 \\
\hline
\end{tabular}

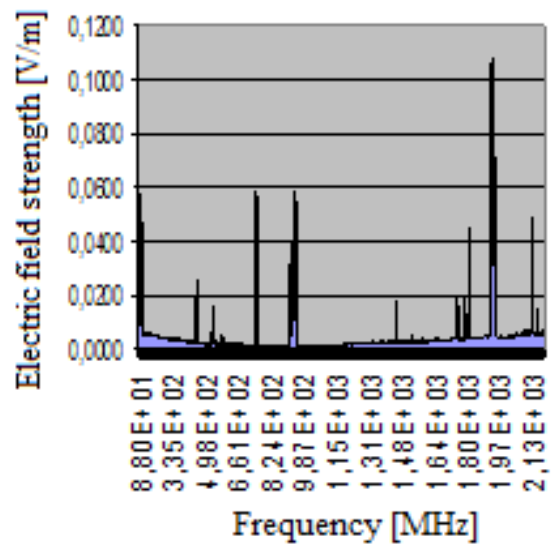

Fig. 2. Ambient noise measurements at 11.36 (background 2, point 1)

The graph is based on the following measurements - Table 2.

\begin{tabular}{|l|l|l|}
\hline $\begin{array}{c}\text { Frequency } \\
\mathrm{MHz}\end{array}$ & $\begin{array}{l}\text { Field Strength } \\
\mathrm{V} / \mathrm{m}\end{array}$ & $\begin{array}{l}\text { Power Density } \\
\mu \mathrm{W} / \mathrm{cm} 2\end{array}$ \\
\hline $1,95 \mathrm{E}+03$ & 0,1079 & 0,0031 \\
\hline $1,93 \mathrm{E}+03$ & 0,1065 & 0,0030 \\
\hline $1,95 \mathrm{E}+03$ & 0,0933 & 0,0023 \\
\hline $1,94 \mathrm{E}+03$ & 0,0864 & 0,0020 \\
\hline $1,95 \mathrm{E}+03$ & 0,0832 & 0,0018 \\
\hline $1,93 \mathrm{E}+03$ & 0,0782 & 0,0016 \\
\hline $1,95 \mathrm{E}+03$ & 0,0714 & 0,0014 \\
\hline $1,96 \mathrm{E}+03$ & 0,0706 & 0,0013 \\
\hline $1,95 \mathrm{E}+03$ & 0,0693 & 0,0013 \\
\hline $1,95 \mathrm{E}+03$ & 0,0681 & 0,0012 \\
\hline $1,94 \mathrm{E}+03$ & 0,0642 & 0,0011 \\
\hline $1,94 \mathrm{E}+03$ & 0,0630 & 0,0011 \\
\hline $1,96 \mathrm{E}+03$ & 0,0604 & 0,0010 \\
\hline $1,96 \mathrm{E}+03$ & 0,0594 & 0,0009 \\
\hline
\end{tabular}

\begin{tabular}{|l|l|l|}
\hline $9,59 \mathrm{E}+02$ & 0,0589 & 0,0009 \\
\hline $1,04 \mathrm{E}+02$ & 0,0581 & 0,0009 \\
\hline
\end{tabular}

The chart presented in figure 3 is based on the following measurements -Table 3.

\begin{tabular}{|l|l|l|}
\hline $\begin{array}{l}\text { Frequency } \\
\mathrm{MHz}\end{array}$ & $\begin{array}{l}\text { Field Strength } \\
\mathrm{V} / \mathrm{m}\end{array}$ & $\begin{array}{l}\text { Power Density } \\
\mu \mathrm{W} / \mathrm{cm} 2\end{array}$ \\
\hline $1,95 \mathrm{E}+03$ & 0,1094 & 0,0032 \\
\hline $1,96 \mathrm{E}+03$ & 0,1084 & 0,0031 \\
\hline $1,94 \mathrm{E}+03$ & 0,1020 & 0,0028 \\
\hline $1,95 \mathrm{E}+03$ & 0,0965 & 0,0025 \\
\hline $1,93 \mathrm{E}+03$ & 0,0892 & 0,0021 \\
\hline $1,94 \mathrm{E}+03$ & 0,0832 & 0,0018 \\
\hline $1,96 \mathrm{E}+03$ & 0,0824 & 0,0018 \\
\hline $1,96 \mathrm{E}+03$ & 0,0822 & 0,0018 \\
\hline $1,94 \mathrm{E}+03$ & 0,0780 & 0,0016 \\
\hline $7,59 \mathrm{E}+02$ & 0,0780 & 0,0016 \\
\hline $1,94 \mathrm{E}+03$ & 0,0775 & 0,0016 \\
\hline $1,93 \mathrm{E}+03$ & 0,0774 & 0,0016 \\
\hline $1,95 \mathrm{E}+03$ & 0,0758 & 0,0015 \\
\hline $1,94 \mathrm{E}+03$ & 0,0746 & 0,0015 \\
\hline $1,91 \mathrm{E}+02$ & 0,0740 & 0,0015 \\
\hline $1,94 \mathrm{E}+03$ & 0,0731 & 0,0014 \\
\hline
\end{tabular}

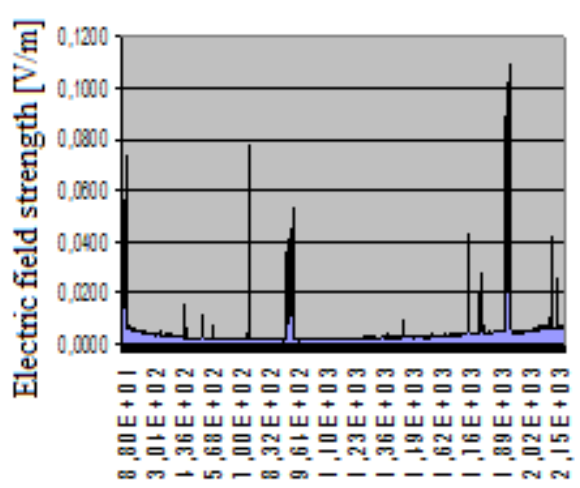

Frequency $[\mathrm{MHz}]$

Fig.3. Ambient noise measurements at 14.41 (background 3, point1)

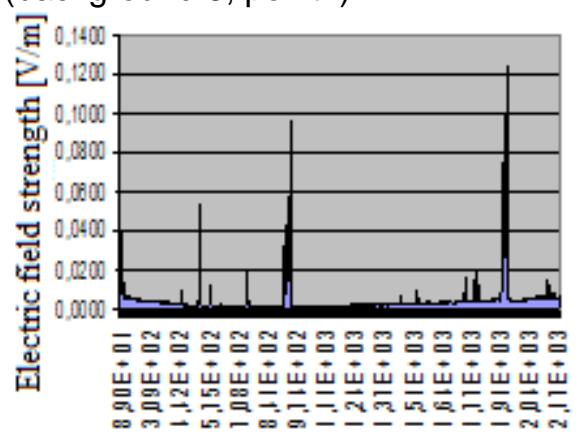

Frequency $[\mathrm{MHz}]$

Fig. 4. Ambient noise measurements at 15.54 (background 4, point 1- below aft deck)

The above chart is based on the measurements listed below - Table 4. 
"Mircea cel Batran" Naval Academy Scientific Bulletin, Volume XIX - 2016 - Issue 1

Published by "Mircea cel Batran" Naval Academy Press, Constanta, Romania // The journal is indexed in:

PROQUEST / DOAJ / DRJI / JOURNAL INDEX / I2OR / SCIENCE LIBRARY INDEX / Google Scholar / Crossref /

Academic Keys I ROAD Open Access / OAJI / Academic Resources / Scientific Indexing Services / SCIPIO

\begin{tabular}{|l|l|l|}
\hline $\begin{array}{l}\text { Frequency } \\
\mathrm{MHz}\end{array}$ & $\begin{array}{l}\text { Field Strength } \\
\mathrm{V} / \mathrm{m}\end{array}$ & $\begin{array}{l}\text { Power Density } \\
\mu \mathrm{W} / \mathrm{cm} 2\end{array}$ \\
\hline $1,96 \mathrm{E}+03$ & 0,1246 & 0,0041 \\
\hline $1,95 \mathrm{E}+03$ & 0,1004 & 0,0027 \\
\hline $9,59 \mathrm{E}+02$ & 0,0961 & 0,0024 \\
\hline $1,96 \mathrm{E}+03$ & 0,0938 & 0,0023 \\
\hline $1,95 \mathrm{E}+03$ & 0,0925 & 0,0023 \\
\hline $1,95 \mathrm{E}+03$ & 0,0803 & 0,0017 \\
\hline $9,60 \mathrm{E}+02$ & 0,0753 & 0,0015 \\
\hline $1,93 \mathrm{E}+03$ & 0,0750 & 0,0015 \\
\hline $1,94 \mathrm{E}+03$ & 0,0744 & 0,0015 \\
\hline $1,95 \mathrm{E}+03$ & 0,0689 & 0,0013 \\
\hline $1,95 \mathrm{E}+03$ & 0,0644 & 0,0011 \\
\hline $1,96 \mathrm{E}+03$ & 0,0634 & 0,0011 \\
\hline $1,93 \mathrm{E}+03$ & 0,0612 & 0,0010 \\
\hline $1,94 \mathrm{E}+03$ & 0,0589 & 0,0009 \\
\hline $9,52 \mathrm{E}+02$ & 0,0580 & 0,0009 \\
\hline $1,94 \mathrm{E}+03$ & 0,0578 & 0,0009 \\
\hline
\end{tabular}

\begin{tabular}{|l|l|l|}
\hline $9,37 \mathrm{E}+02$ & 0,0317 & 0,0003 \\
\hline
\end{tabular}

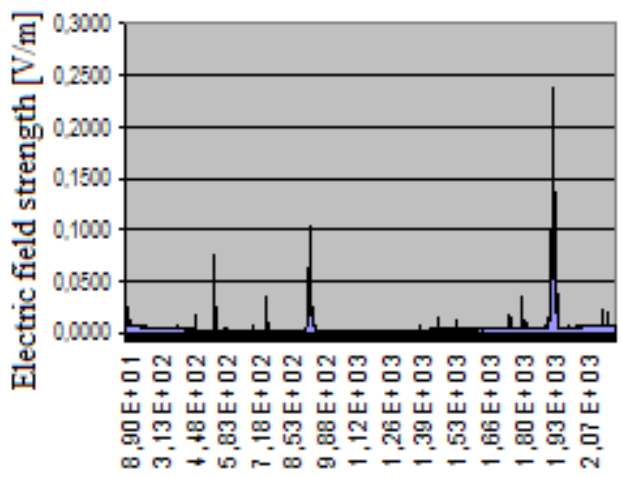

Frequency $[\mathrm{MHz}]$

Fig. 6. Ambient noise measurements at 17.05 (background 6, point 3)

The graph is based on the following measurements - Table 6.

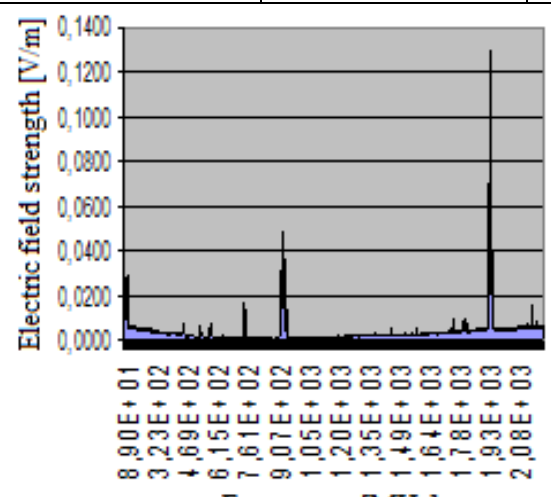

Frequency $[\mathrm{MHz}]$

Fig. 5. Ambient noise measurements at 16.42 (background 5, point 2)

Figure 5 is based on the following measurements -Table 5.

\begin{tabular}{|l|l|l|}
\hline $\begin{array}{l}\text { Frequency } \\
\mathrm{MHz}\end{array}$ & $\begin{array}{l}\text { Field Strength } \\
\mathrm{V} / \mathrm{m}\end{array}$ & $\begin{array}{l}\text { Power Density } \\
\mu \mathrm{W} / \mathrm{cm} 2\end{array}$ \\
\hline $1,95 \mathrm{E}+03$ & 0,1301 & 0,0045 \\
\hline $1,95 \mathrm{E}+03$ & 0,1123 & 0,0033 \\
\hline $1,94 \mathrm{E}+03$ & 0,0918 & 0,0022 \\
\hline $1,94 \mathrm{E}+03$ & 0,0902 & 0,0022 \\
\hline $1,94 \mathrm{E}+03$ & 0,0854 & 0,0019 \\
\hline $1,95 \mathrm{E}+03$ & 0,0821 & 0,0018 \\
\hline $1,95 \mathrm{E}+03$ & 0,0647 & 0,0011 \\
\hline $1,94 \mathrm{E}+03$ & 0,0504 & 0,0007 \\
\hline $9,44 \mathrm{E}+02$ & 0,0487 & 0,0006 \\
\hline $1,94 \mathrm{E}+03$ & 0,0437 & 0,0005 \\
\hline $1,96 \mathrm{E}+03$ & 0,0406 & 0,0004 \\
\hline $1,94 \mathrm{E}+03$ & 0,0397 & 0,0004 \\
\hline $9,45 \mathrm{E}+02$ & 0,0359 & 0,0003 \\
\hline $1,93 \mathrm{E}+03$ & 0,0334 & 0,0003 \\
\hline $1,96 \mathrm{E}+03$ & 0,0320 & 0,0003 \\
\hline
\end{tabular}

\begin{tabular}{|l|l|l|}
\hline $\begin{array}{l}\text { Frequency } \\
\mathrm{MHz}\end{array}$ & $\begin{array}{l}\text { Field Strength } \\
\mathrm{V} / \mathrm{m}\end{array}$ & $\begin{array}{l}\text { Power Density } \\
\mu \mathrm{W} / \mathrm{cm} 2\end{array}$ \\
\hline $5,43 \mathrm{E}+02$ & 0,0769 & 0,0016 \\
\hline $9,45 \mathrm{E}+02$ & 0,1031 & 0,0028 \\
\hline $9,46 \mathrm{E}+02$ & 0,0955 & 0,0024 \\
\hline $1,95 \mathrm{E}+03$ & 0,1115 & 0,0033 \\
\hline $1,93 \mathrm{E}+03$ & 0,0859 & 0,0020 \\
\hline $1,95 \mathrm{E}+03$ & 0,1365 & 0,0049 \\
\hline $1,95 \mathrm{E}+03$ & 0,1324 & 0,0047 \\
\hline $1,94 \mathrm{E}+03$ & 0,1049 & 0,0029 \\
\hline $1,94 \mathrm{E}+03$ & 0,1633 & 0,0071 \\
\hline $1,94 \mathrm{E}+03$ & 0,1571 & 0,0065 \\
\hline $1,94 \mathrm{E}+03$ & 0,0909 & 0,0022 \\
\hline $1,94 \mathrm{E}+03$ & 0,2383 & 0,0151 \\
\hline $1,94 \mathrm{E}+03$ & 0,0870 & 0,0020 \\
\hline $1,94 \mathrm{E}+03$ & 0,2129 & 0,0120 \\
\hline $1,94 \mathrm{E}+03$ & 0,1070 & 0,0030 \\
\hline $1,93 \mathrm{E}+03$ & 0,1039 & 0,0029 \\
\hline
\end{tabular}

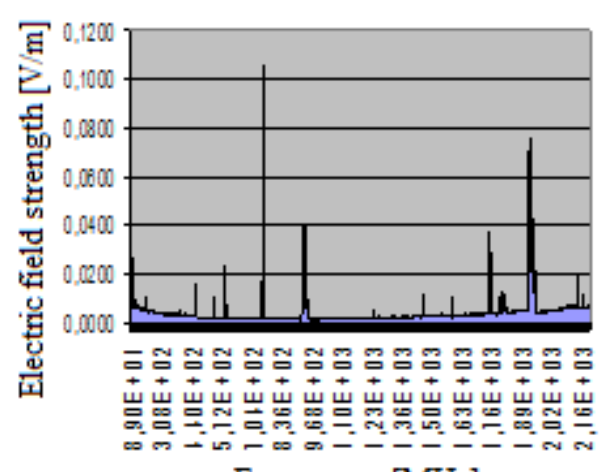

Frequency $[\mathrm{MHz}]$

Fig. 7. Ambient noise measurements at 17.44 (background 7, point 4) 
"Mircea cel Batran" Naval Academy Scientific Bulletin, Volume XIX - 2016 - Issue 1

Published by "Mircea cel Batran" Naval Academy Press, Constanta, Romania // The journal is indexed in:

PROQUEST / DOAJ / DRJI / JOURNAL INDEX / I2OR / SCIENCE LIBRARY INDEX / Google Scholar / Crossref /

Academic Keys / ROAD Open Access / OAJI / Academic Resources / Scientific Indexing Services / SCIPIO

Figure 7 is based on the following measurements -Table 7.

\begin{tabular}{|l|l|l|}
\hline $\begin{array}{l}\text { Frequency } \\
\mathrm{MHz}\end{array}$ & $\begin{array}{l}\text { Field Strength } \\
\mathrm{V} / \mathrm{m}\end{array}$ & $\begin{array}{l}\text { Power Density } \\
\mu \mathrm{W} / \mathrm{cm} 2\end{array}$ \\
\hline $9,70 \mathrm{E}+01$ & 0,0349 & 0,0003 \\
\hline $9,80 \mathrm{E}+01$ & 0,0412 & 0,0004 \\
\hline $7,59 \mathrm{E}+02$ & 0,1067 & 0,0030 \\
\hline $7,60 \mathrm{E}+02$ & 0,0761 & 0,0015 \\
\hline $9,42 \mathrm{E}+02$ & 0,0402 & 0,0004 \\
\hline $1,76 \mathrm{E}+03$ & 0,0375 & 0,0004 \\
\hline $1,93 \mathrm{E}+03$ & 0,0707 & 0,0013 \\
\hline $1,93 \mathrm{E}+03$ & 0,0708 & 0,0013 \\
\hline $1,94 \mathrm{E}+03$ & 0,0413 & 0,0005 \\
\hline $1,94 \mathrm{E}+03$ & 0,0499 & 0,0007 \\
\hline $1,94 \mathrm{E}+03$ & 0,0410 & 0,0004 \\
\hline $1,94 \mathrm{E}+03$ & 0,0386 & 0,0004 \\
\hline $1,94 \mathrm{E}+03$ & 0,0677 & 0,0012 \\
\hline $1,94 \mathrm{E}+03$ & 0,0761 & 0,0015 \\
\hline $1,94 \mathrm{E}+03$ & 0,0433 & 0,0005 \\
\hline $1,95 \mathrm{E}+03$ & 0,0425 & 0,0005 \\
\hline & &
\end{tabular}

2.2. Electromagnetic power spectral density representation, with station 3 in broadcasting $(250 \mathrm{MHz})$, on the ship - below the helipad deck and in the harbor area

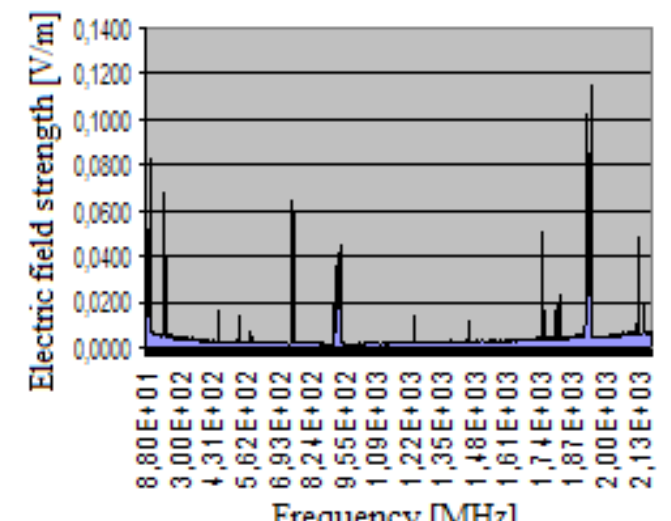

Frequency $[\mathrm{MHz}]$

Fig. 8. Ambient noise measurements at 14:47 (point 1). The station broadcasts unmodulated at $250 \mathrm{MHz}$.

The chart is based on the following measurements -Table 8.

\begin{tabular}{|l|l|l|}
\hline $\begin{array}{l}\text { Frequency } \\
\mathrm{MHz}\end{array}$ & $\begin{array}{l}\text { Field Strength } \\
\mathrm{V} / \mathrm{m}\end{array}$ & $\begin{array}{l}\text { Power Density } \\
\mu \mathrm{W} / \mathrm{cm} 2\end{array}$ \\
\hline $1,96 \mathrm{E}+03$ & 0,1147 & 0,0035 \\
\hline $1,93 \mathrm{E}+03$ & 0,1029 & 0,0028 \\
\hline $1,94 \mathrm{E}+03$ & 0,0961 & 0,0024 \\
\hline $1,96 \mathrm{E}+03$ & 0,0926 & 0,0023 \\
\hline $1,96 \mathrm{E}+03$ & 0,0916 & 0,0022 \\
\hline $1,93 \mathrm{E}+03$ & 0,0869 & 0,0020 \\
\hline $1,96 \mathrm{E}+03$ & 0,0866 & 0,0020 \\
\hline
\end{tabular}

(c) 2015. This work is licensed under the Creative Commons Attribution-Noncommercial-Share Alike 4.0 License.

\begin{tabular}{|l|l|l|}
\hline $1,94 \mathrm{E}+03$ & 0,0846 & 0,0019 \\
\hline $1,91 \mathrm{E}+02$ & 0,0827 & 0,0018 \\
\hline $1,96 \mathrm{E}+03$ & 0,0753 & 0,0015 \\
\hline $1,95 \mathrm{E}+03$ & 0,0731 & 0,0014 \\
\hline $1,92 \mathrm{E}+02$ & 0,0729 & 0,0014 \\
\hline $1,94 \mathrm{E}+03$ & 0,0715 & 0,0014 \\
\hline $1,93 \mathrm{E}+03$ & 0,0711 & 0,0013 \\
\hline $1,94 \mathrm{E}+03$ & 0,0711 & 0,0013 \\
\hline $2,50 \mathrm{E}+02$ & 0,0683 & 0,0012 \\
\hline $7,60 \mathrm{E}+02$ & 0,0653 & 0,0011 \\
\hline $1,94 \mathrm{E}+03$ & 0,0609 & 0,0010 \\
\hline $1,95 \mathrm{E}+03$ & 0,0603 & 0,0010 \\
\hline
\end{tabular}

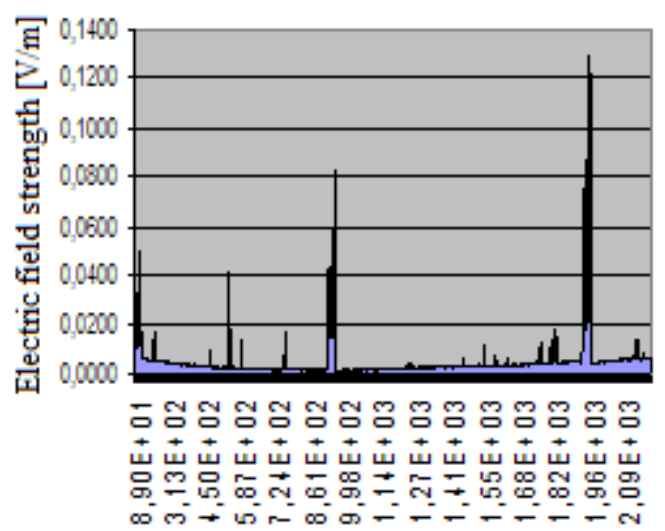

Frequency $[\mathrm{MHz}]$

Fig. 9. Ambient noise measurements at 15:57 (point 1). The station broadcasts unmodulated at $250 \mathrm{MHz}$.

Figure 9 was drawn using the values from Table 9.

\begin{tabular}{|l|l|l|}
\hline $\begin{array}{l}\text { Frequency } \\
\mathrm{MHz}\end{array}$ & $\begin{array}{l}\text { Field Strength } \\
\mathrm{V} / \mathrm{m}\end{array}$ & $\begin{array}{l}\text { Power Density } \\
\mu \mathrm{W} / \mathrm{cm} 2\end{array}$ \\
\hline $1,96 \mathrm{E}+03$ & 0,1293 & 0,1293 \\
\hline $1,95 \mathrm{E}+03$ & 0,1084 & 0,1084 \\
\hline $1,96 \mathrm{E}+03$ & 0,0957 & 0,0957 \\
\hline $1,96 \mathrm{E}+03$ & 0,0928 & 0,0928 \\
\hline $1,95 \mathrm{E}+03$ & 0,0871 & 0,0871 \\
\hline $9,59 \mathrm{E}+02$ & 0,0827 & 0,0827 \\
\hline $1,93 \mathrm{E}+03$ & 0,0756 & 0,0756 \\
\hline $1,95 \mathrm{E}+03$ & 0,0704 & 0,0704 \\
\hline $1,96 \mathrm{E}+03$ & 0,0680 & 0,0680 \\
\hline $1,93 \mathrm{E}+03$ & 0,0647 & 0,0647 \\
\hline $1,95 \mathrm{E}+03$ & 0,0633 & 0,0633 \\
\hline $9,60 \mathrm{E}+02$ & 0,0622 & 0,0622 \\
\hline $1,94 \mathrm{E}+03$ & 0,0611 & 0,0611 \\
\hline $9,53 \mathrm{E}+02$ & 0,0590 & 0,0590 \\
\hline $1,94 \mathrm{E}+03$ & 0,0589 & 0,0589 \\
\hline $2,50 \mathrm{E}+02$ & 0,0170 & 0,0001 \\
\hline
\end{tabular}


"Mircea cel Batran" Naval Academy Scientific Bulletin, Volume XIX - 2016 - Issue 1

Published by "Mircea cel Batran" Naval Academy Press, Constanta, Romania // The journal is indexed in:

PROQUEST / DOAJ / DRJI / JOURNAL INDEX / I2OR / SCIENCE LIBRARY INDEX / Google Scholar / Crossref /

Academic Keys / ROAD Open Access / OAJI / Academic Resources / Scientific Indexing Services / SCIPIO

Figure 10 is based on the measurements from the following table -Table 10.

\begin{tabular}{|l|l|l|}
\hline $\begin{array}{l}\text { Frequency } \\
\mathrm{MHz}\end{array}$ & $\begin{array}{l}\text { Field Strength } \\
\mathrm{V} / \mathrm{m}\end{array}$ & $\begin{array}{l}\text { Power } \\
\text { Density } \\
\mu \mathrm{W} / \mathrm{cm} 2\end{array}$ \\
\hline $1,95 \mathrm{E}+03$ & 0,1383 & 0,0051 \\
\hline $1,95 \mathrm{E}+03$ & 0,0969 & 0,0025 \\
\hline $1,94 \mathrm{E}+03$ & 0,0956 & 0,0024 \\
\hline $1,94 \mathrm{E}+03$ & 0,0848 & 0,0019 \\
\hline $1,95 \mathrm{E}+03$ & 0,0757 & 0,0015 \\
\hline $2,50 \mathrm{E}+02$ & 0,0737 & 0,0014 \\
\hline $1,94 \mathrm{E}+03$ & 0,0723 & 0,0014 \\
\hline $1,94 \mathrm{E}+03$ & 0,0644 & 0,0011 \\
\hline $1,95 \mathrm{E}+03$ & 0,0643 & 0,0011 \\
\hline $1,94 \mathrm{E}+03$ & 0,0577 & 0,0009 \\
\hline $1,94 \mathrm{E}+03$ & 0,0571 & 0,0009 \\
\hline $1,96 \mathrm{E}+03$ & 0,0556 & 0,0008 \\
\hline $9,37 \mathrm{E}+02$ & 0,0547 & 0,0008 \\
\hline $2,51 \mathrm{E}+02$ & 0,0546 & 0,0008 \\
\hline $1,94 \mathrm{E}+03$ & 0,0527 & 0,0007 \\
\hline $1,96 \mathrm{E}+03$ & 0,0508 & 0,0007 \\
\hline
\end{tabular}

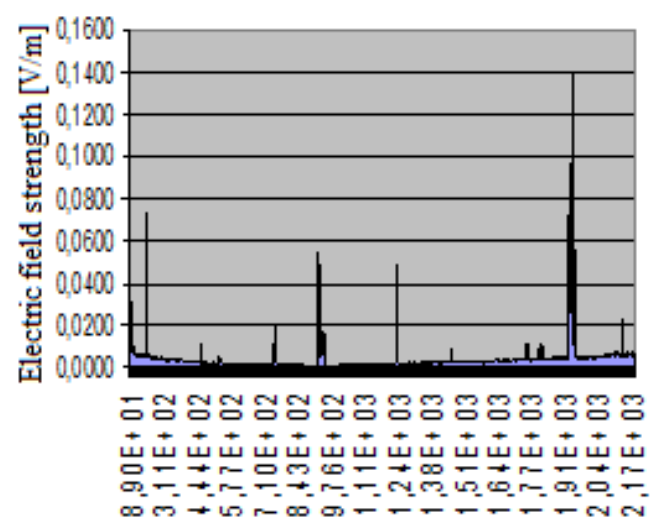

Frequency $[\mathrm{MHz}]$

Fig. 10. Ambient noise measurements at 16:46 (point 2). The station broadcasts unmodulated at $250 \mathrm{MHz}$.

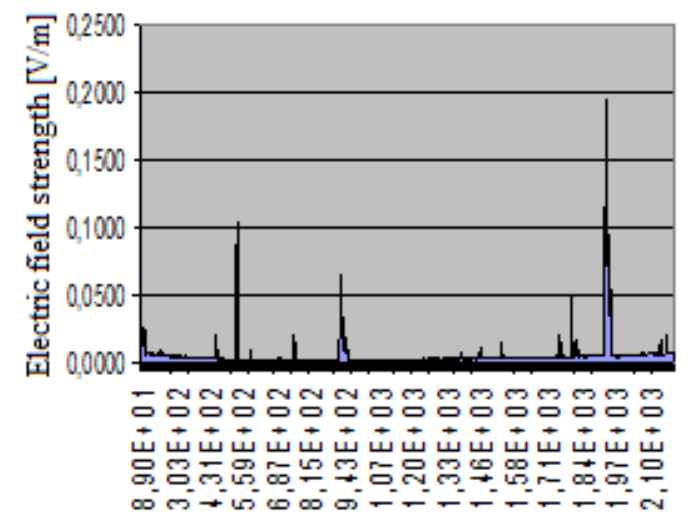

Frequency $[\mathrm{MHz}]$
Fig. 11. Ambient noise measurements at 17:08 (point 3 - at approximate 150m from The Green Lighthouse). The military station number 3 broadcasts unmodulated at $250 \mathrm{MHz}$.

Chart 11 is based on the measurements from table 11.

\begin{tabular}{|l|l|l|}
\hline $\begin{array}{l}\text { Frequency } \\
\mathrm{MHz}\end{array}$ & $\begin{array}{l}\text { Field Strength } \\
\mathrm{V} / \mathrm{m}\end{array}$ & $\begin{array}{l}\text { Power Density } \\
\mu \mathrm{W} / \mathrm{cm} 2\end{array}$ \\
\hline $1,94 \mathrm{E}+03$ & 0,1947 & 0,0101 \\
\hline $1,94 \mathrm{E}+03$ & 0,1475 & 0,0058 \\
\hline $1,94 \mathrm{E}+03$ & 0,1407 & 0,0053 \\
\hline $1,94 \mathrm{E}+03$ & 0,1373 & 0,0050 \\
\hline $1,94 \mathrm{E}+03$ & 0,1205 & 0,0038 \\
\hline $1,93 \mathrm{E}+03$ & 0,1158 & 0,0036 \\
\hline $1,94 \mathrm{E}+03$ & 0,1149 & 0,0035 \\
\hline $1,94 \mathrm{E}+03$ & 0,1102 & 0,0032 \\
\hline $1,94 \mathrm{E}+03$ & 0,1089 & 0,0031 \\
\hline $5,44 \mathrm{E}+02$ & 0,1028 & 0,0028 \\
\hline $1,95 \mathrm{E}+03$ & 0,0954 & 0,0024 \\
\hline $1,95 \mathrm{E}+03$ & 0,0884 & 0,0021 \\
\hline $1,93 \mathrm{E}+03$ & 0,0851 & 0,0019 \\
\hline $1,94 \mathrm{E}+03$ & 0,0832 & 0,0018 \\
\hline $5,43 \mathrm{E}+02$ & 0,0824 & 0,0018 \\
\hline $2,50 \mathrm{E}+02$ & 0,0098 & 0,0098 \\
\hline
\end{tabular}

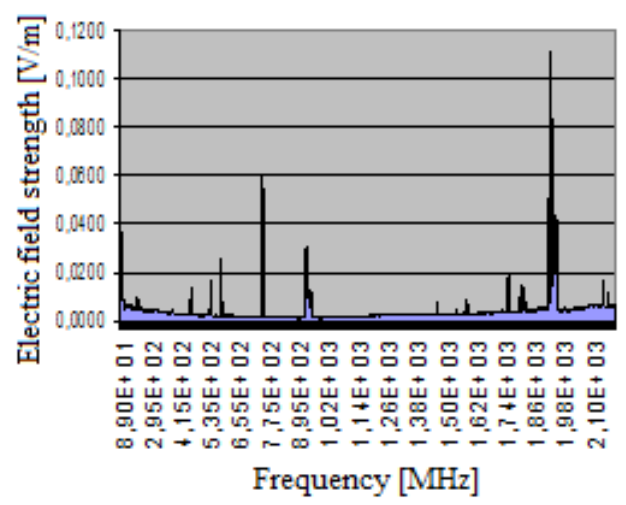

Fig. 12. Ambient noise measurements at 17:47 (point 4 - Berth 30). The station broadcasts unmodulated at $250 \mathrm{MHz}$.

Chart 12 is based on the measurements from table 12.

\begin{tabular}{|l|l|l|}
\hline $\begin{array}{l}\text { Frequency } \\
\mathrm{MHz}\end{array}$ & $\begin{array}{l}\text { Field Strength } \\
\mathrm{V} / \mathrm{m}\end{array}$ & $\begin{array}{l}\text { Power Density } \\
\mu \mathrm{W} / \mathrm{cm} 2\end{array}$ \\
\hline $1,93 \mathrm{E}+03$ & 0,1109 & 0,0033 \\
\hline $1,93 \mathrm{E}+03$ & 0,1100 & 0,0032 \\
\hline $1,94 \mathrm{E}+03$ & 0,0829 & 0,0018 \\
\hline $1,94 \mathrm{E}+03$ & 0,0642 & 0,0011 \\
\hline $1,93 \mathrm{E}+03$ & 0,0623 & 0,0010 \\
\hline $7,60 \mathrm{E}+02$ & 0,0608 & 0,0010 \\
\hline $7,59 \mathrm{E}+02$ & 0,0590 & 0,0009 \\
\hline $7,65 \mathrm{E}+02$ & 0,0546 & 0,0008 \\
\hline $1,93 \mathrm{E}+03$ & 0,0519 & 0,0007 \\
\hline
\end{tabular}

295 
"Mircea cel Batran" Naval Academy Scientific Bulletin, Volume XIX - 2016 - Issue 1 Published by "Mircea cel Batran" Naval Academy Press, Constanta, Romania // The journal is indexed in: PROQUEST / DOAJ / DRJI / JOURNAL INDEX / I2OR / SCIENCE LIBRARY INDEX / Google Scholar / Crossref /

Academic Keys / ROAD Open Access / OAJI / Academic Resources / Scientific Indexing Services / SCIPIO

\begin{tabular}{|l|l|l|}
\hline $1,93 \mathrm{E}+03$ & 0,0511 & 0,0007 \\
\hline $1,94 \mathrm{E}+03$ & 0,0479 & 0,0006 \\
\hline $1,94 \mathrm{E}+03$ & 0,0475 & 0,0006 \\
\hline $1,94 \mathrm{E}+03$ & 0,0474 & 0,0006 \\
\hline $1,93 \mathrm{E}+03$ & 0,0458 & 0,0006 \\
\hline
\end{tabular}

\begin{tabular}{|l|l|l|}
\hline $1,95 \mathrm{E}+03$ & 0,0430 & 0,0005 \\
\hline $2,50 \mathrm{E}+02$ & 0,0102 & 0,0000 \\
\hline
\end{tabular}

\section{CONCLUSIONS}

From the data obtained by measurements it can be observed, that in the measured frequency range ( 80 $\mathrm{MHz}-2,2 \mathrm{GHz}$ ), the radiated power densities and the electric field strength values do not exceed the basic restrictions for health protection, both for European standards (EN 60215: 1994 Safety Requirements for Radio Transmitting Equipment, En 50371) and American rules (FCC's Rule Parts 1. 1310, 2.1091 and 2.1093). The radio stations that work in the frequency ranges that are covered by the measurements issue small value powers. For example, the power standard for the civil stations that work between $156-174 \mathrm{MHz}$ (VHF maritime) is $1 \mathrm{~W}$ inside the harbour area and $25 \mathrm{~W}$ outside. Thus, the maximum measured value for the electric field strength is not exceeding $1 \mathrm{~V} / \mathrm{m}$, and for the electromagnetic power density the value of 0,01 $\mu \mathrm{W} / \mathrm{cm}^{2}$. The European Standard provides a level of $28 \mathrm{~V} / \mathrm{m}$ to $200 \mu \mathrm{W} / \mathrm{cm}^{2}$ in the range $10-400 \mathrm{MHz}$, respectively $1375 \cdot \sqrt{f}$ and $\frac{f \cdot 10^{2}}{200} \mu \mathrm{W} / \mathrm{cm}^{2}$ in the frequency range of $0.4-2 \mathrm{GHz}$. The American Rule provides:

- a level of $27,5 \mathrm{~V} / \mathrm{m}$ and $200 \mu \mathrm{W} / \mathrm{cm}^{2}$ in the range of $30-300 \mathrm{MHz}$; respectively $\frac{f \cdot 10^{3}}{1500} \mu \mathrm{W} / \mathrm{cm}^{2}$ in the range of $300-400 \mathrm{MHz}$ and $0,4-1,5 \mathrm{GHz}$; respectively $1 \mathrm{~mW} / \mathrm{cm}^{2}$ in the range of $1,5-2 \mathrm{GHz}$;

Due to the lack of measurement sensors for the analyzer used in frequency range below $80 \mathrm{MHz}$ and higher than $2 \mathrm{GHz}$ no measurements were performed in frequency ranges with strong signal sources. For example, for the radio stations, in the range of 4-30 MHz (HF maritime) the emission power is between 400-750 W and the special stations use power up to $1.5 \mathrm{~kW}$. For the radiolocation stations the emission powers for 9 $\mathrm{GHz}$ are 15 and $25 \mathrm{~kW}$. Thus, if we extrapolate the value of the electric field strength radiated by the radio station with the power of 25 watts $(0.1324 \mathrm{~V} / \mathrm{m})$ for $25 \mathrm{~kW}$ emission power one can a value of $132.4 \mathrm{~V} / \mathrm{m}$ for the radiated electric field strength. Such value is dangerous for the human factor.

\section{BIBLIOGRAPHY}

[1] ${ }^{\star \star \star}$ - Ecologie electromagnetică-caracterizarea surselor, diagnosticarea efectelor-prevenirea şi combaterea lor-Contract 6115 CEEX, 2005Mataluno

[2] Calotă, S.V., Deliu, N., Licurici, M., Ferastraeru, C., Contulescu, A., Studiu privind expunerea profesională la radiaţii neionizante de înaltă frecvenţă şi riscul pentru sănătate, Institutul de Sănătate Publică, Bucureşti, 1998

[3] lanoz, M.(Profesor la Universitatea Politehnică din Lausanne, Elveţia), Biological and Health Effects of Electromagnetic Fields, conferinţă, Universitatea Politehnica, Bucureşti, Fac. de Energetică, Centrul de cercetare TICEM , martie 2003

[4] Zamfirescu, M., Sajin, I., Rusu, I., Sajin, M., Kovacs, E., Efecte biologice ale radiaţiilor electromagnetice de radiofrecvenţă şi microunde, Editura Medicală, Bucureşti, 2000

[5] Mataluno G., Le radiozioni elettromagnetiche a bordo, în Rivista marittima, anno CXXI - Dicembre, Roma, 1988

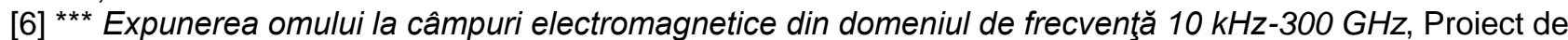
Standard Român: SR EN 50166-2, IRS, Bucureşti (conform cu standardul EN 50166-2/1994)

[7] *** Hotărârea Guvernului României, HG nr. 59, din 06.09.2006, privind cerinţele minime de securitate şi sănătate referitoare la expunerea lucrătorilor la riscuri generate de câmpurile electromagnetice - Anexă: Valori limită de expunere şi valori de declanşare a acțiunii pentru câmpurile electromagnetice;

[8] *** Directiva Specifică 96/98/EC, referitoare la „Echipamentul maritim”, obligatorie de la 1 ianuarie 1999, transpusă în România prin Ordinul ministrului lucrărilor publice, transporturilor şi locuinţei nr. 582/2003, pentru aprobarea normelor tehnice de tip a echipamentelor şi produselor pentru nave maritime, prevăzute de convenţiile internaţionale la care România este parte, cod MLPLTL.ANR-EM 2003;

[9] *** Standardul românesc SREN 60945:2001 - Echipamente şi sisteme de navigaţie şi radiocomunicaţii maritime. Reguli generale. Metode de încercare şi rezultate impuse; 
"Mircea cel Batran" Naval Academy Scientific Bulletin, Volume XIX - 2016 - Issue 1

Published by "Mircea cel Batran" Naval Academy Press, Constanta, Romania /I The journal is indexed in: PROQUEST I DOAJ / DRJI / JOURNAL INDEX I I2OR / SCIENCE LIBRARY INDEX / Google Scholar / Crossref I Academic Keys / ROAD Open Access / OAJI / Academic Resources / Scientific Indexing Services / SCIPIO

[10] ${ }^{* * *}$ Analiza comparativă a normelor europene şi americane cu privire la limitele de expunere la câmp electromagnetic a organismului uman în gama de frecvenţa $\mathrm{O} \mathrm{Hz} \mathrm{la} 300 \mathrm{GHz}$, lucrarea „Radio-Freqnency Radiation for Transmitters: A Comparison of U.S. and European Requirements”, autori: Steve Dillingham şi Nick Cobb);

[11] ***Standardele româneşti SREN 50166-1 şi SREN 50166-2, referitoare la limitele admise ale densităţii curentului indus şi efectele biologice asociate, limitele ratei specifice de absorbţie - SAR, limitele admise ale intensităţii câmpului electric ale celui magnetic şi ale densităţii de putere de vârf în corpul omenesc, pentru medii controlate şi necontrolate, în gama de frecvenţă $3 \mathrm{kHz}-300 \mathrm{GHz}$; 